\title{
MF_CSA AI based Controlling Technique for Single Phase Converter Output Enhancement at Different Loading Conditions
}

\author{
Manisha Patel \\ M. Tech Scholar \\ Technocrats Institute of Technology \\ Bhopal, (MP), India \\ manupatel886@gmail.com
}

\author{
Dr Malaya Saurava Das \\ Associate Professor \\ Technocrats Institute of Technology \\ Bhopal (MP) India \\ malaya_rec@rediffmail.com
}

\begin{abstract}
Renewable and sustainable sources such as SPV systems and WT energy conversion systems are now seen as a promising and growing alternative energy source in view of global emissions and deterioration of the FP. the main objective of the study designing of a solar PV system with varying irradiation in MATLAB/SIMULINK and enhance its output capacity before its integration with the grid. Also, the system has to be made to drive an unbalanced load and dynamic load so that it is capable of handling the change in the power demand of the system. And to design a suitable controller for the multilevel power converter such that it produces better output results than the traditional converter. AI technique has to be incorporated in the converter designing. This work provides a comprehensive design and implementation of power regulatory per phase inverter with proposed MF-CSA (Multi-Function Crow Search Algorithm) optimization controller. The Inverter has been provided with a proposed optimization technique while integrating it with the grid. The voltage output of the system from the modeled solar system with varying irradiation and temperature control is being fed to the inverter for $\mathrm{DC}$ to $\mathrm{AC}$ conversion.
\end{abstract}

Keywords: DC, AC, CSA, SPS.

\section{INTRODUCTION}

Renewable and sustainable sources such as SPV systems and WT energy conversion systems are now seen as a promising and growing alternative energy source in view of global emissions and deterioration of the FP [1]. These problems of non-renewable sources can be significantly reduced by increasing the use of renewable sources in DERs and MGs. These renewable sources have different characteristics such as the generation of electricity without emissions, they are eternal and, above all, the fuels used to generate electricity from solar and wind energy, are solar radiation and moving air masses, abundant and also freely available. . Previous studies have also shown that the stability of the traditional electricity grid can also be improved by integrating renewable sources into the electricity grid through MG and DER.
In conventional power systems, electricity is produced at the other end of the load and this electricity is carried from the source to the load over a long transmission network, resulting in increased transmission and distribution losses. These losses can be greatly reduced by producing electricity after charging is complete and meeting the charging requirements of the surrounding areas.

DER and MG are considered local power systems for having the aforementioned characteristics that power systems engineers have been looking for for many years. Energy storage and renewable sources used in DERs and MGs are connected to the grid via the Grid Connected Inverter (GCI). However, using an inverter only to supply active current to the grid ultimately increases the capital investment, maintenance costs and man-hours

\section{LITERATURE REVIEW}

Darshni M. Shukla et al. [1] this article presents the multifunctional operation of a three-stage cascaded H-bridge inverter for grid-connected solar photovoltaic applications. Solar panel and inverter are modeled with three control technologies (pq, dq, cpt) for asymmetric and non-linear loads. Performance is simulated in the MATLAB environment using the SIMULINK and Sim Power System (SPS) toolkits. Inverter performance is evaluated for harmonic elimination, power factor correction as well as active and reactive power support for grid and non-linear load.

S. Balasubramanyan et al. [2] Improvement of the problem of energy quality in the electricity grid. Power compensation and optimization for connection to the grid with a photovoltaic system. The effects of reactive power compensation and optimization on the reliability and quality of the system and propose a coordinated distribution of active and reactive power to solve this problem. First, a vector method is developed to illustrate the principle of energy distribution. 
P M Ansho et al. [3] This paper discusses these issues, examines the consequences of reactive power compensation and optimization on system reliability and quality, and suggests coordinated distribution of active and reactive power to deal with this problem. First, a vector method is developed for instance the principle of energy distribution. As a result, the connection between power and voltage is analyzed with a good operating range.

Kangan Wang et al. [4] this paper, a dynamic power distribution strategy has been proposed to eliminate over modulation during a CHB-PV system by properly redistributing reactive power to the inverter modules of the CHB-PV system. The proposed strategy can effectively expand the operational range of the CHB-PV system with an easy control and implementation algorithm. Simulations and experimental results run on a CHB-PV system connected to the seven-stage network are presented to validate the proposed strategy.

\section{OBJECTIVE}

There are following objectives that are to be expected from the work

- Designing of a solar PV system with varying irradiation in MATLAB/SIMULINK and enhance its output capacity before its integration with the grid. Also the system has to be made to drive a unbalanced load and dynamic load so that it is capable of handling the change in the power demand of the system.

- To design a suitable controller for the multilevel power converter such that it produces better output results than the traditional converter. AI technique has to be incorporated in the converter designing.

- The controller has to be designed along with buck boost control of DC output from the system.

- Enhance the system reliability and efficiency by integrating it with the grid via a transformer with the desired grid voltage and frequency.

\section{METHODOLOGY}

The model has been developed in MALAB/SIMULINK environment. It is a matrix / matrix language with control flow instructions, functions, data structures, inputs / outputs and object-oriented programming functions. It has the following main features:

- High level language for scientific and technical computer science

- Desktop environment for exploring, designing and solving iterative problems

- Graphs to display data and tools to create customized graphs
- Applications for curve adaptation, data classification, signal analysis, control optimization and many other tasks

- Complementary toolboxes for various technical and scientific applications

- Tools to create custom applications for the user interface

- Distribution options at no cost for sharing MATLAB programs with end users

The power produced by a single module is seldom enough for commercial use, so modules are connected to form array to supply the load. Connecting modules in an array are the same as connecting cells in a module. The modules can also be connected in series to obtain a higher voltage or in parallel to obtain a higher current.

\section{A. Multi Modular Cell Modeling Control}

The PV farm base distributed generation system consists of four PV arrays at variable irradiation and variable temperature which is considered during modeling. Many factors affect the temperature of the photovoltaic cell: the materials and construction of the module, the mounting and shelf configurations, the incident radiation (modified by shading and pollution), the wind speed at the field level and ambient temperature, among others. The temperature is kept to be varying for different modules from 35 to 45 . Also irradiation is reduced at 0.1 seconds while the simulation takes place for 0.5 second. It is developed as a prototype for the distributed generations at various nearby locations whose output is first combined and then integrated with the grid, and hence reducing the equipment necessities.

\section{B. MF-CSA algorithm}

Traditional research methods have long been used to solve design problems. While these methods produce promising results on many real-world problems, they can fail on more complex design problems. In real design problems, the number of decision variables can be very large and their effect on the objective function can be very complicated. The objective function can have many local optimal, while the designer is interested in the global optimum. These problems cannot be solved by conventional methods which find only local optimal. In these cases, efficient optimization methods are required.

Crows (family of ravens or corvids) are considered to be the most intelligent birds. They contain the largest brain for their size. Based on a brain-to-body ratio, their brains are slightly smaller than that of a human brain. There is a lot of evidence of crows' intelligence. You have demonstrated self-confidence in the mirror test and are able to create tools. Crows can remember faces and warn each other when a rude approaches. Plus, they can use tools, communicate artfully, and remember where their food hides for a few months. 
Initially, a cascade network selection vector control scheme is used to control the inverter-based DG system using two control circuits. In the proposed framework, the objective function is defined in the grid-connected mode, in which the external control function controls the active and reactive power $(\mathrm{P}, \mathrm{Q})$, while the internal function controls the current components of the direct and quadrature (Id, Iq) at constant connection voltage and frequency. . Since metaheuristic algorithms should provide a balance between diversification and intensification, MF-CSA does this by controlling the probability of consciousness (PA) parameter. By lowering the AP value, CSA tends to locate the search area where a good solution is found, thus increasing the intensification and vice versa.

The d-dimensional crows' flock matrix generation: The CSA algorithm starts with the generation of a dimensional matrix with a specified number of crows' (F) i.e (flock size). Each crow in the flock denotes a feasible solution of the problem.

Crows $=\left[\begin{array}{cccc}\mathrm{x}_{1}^{1} & \mathrm{x}_{2}^{1} & \cdots & \mathrm{x}_{\mathrm{d}}^{1} \\ \mathrm{x}_{1}^{2} & \mathrm{x}_{2}^{2} & \cdots & \mathrm{x}_{\mathrm{d}}^{2} \\ \vdots & \cdot & \ddots & \vdots \\ \mathrm{x}_{1}^{\mathrm{F}} & \mathrm{x}_{2}^{\mathrm{F}} & \cdots & \mathrm{x}_{\mathrm{d}}^{\mathrm{F}}\end{array}\right]$

Parameters adjustment: The optimization problem, decision variables and constraints are specified. Then, the adjustable parameters of CSA such as maximum number of iterations (it max), flight length (FL), F, and AP are valued. Here the constraints are decided by calculation of $\mathrm{P}$ and $\mathrm{Q}$ with the $\mathrm{q}$ axis current and voltage value as shown in the flow chart 2 below.

Crow position initialization:

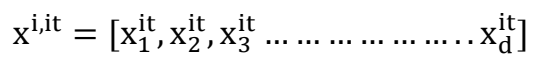

Where, $\mathrm{i}=(1,2 \ldots \mathrm{F})=$ crows' number and $\mathrm{It}=(1,2, \ldots$. , itmax $)$ $=$ iteration number.

Crows memory initialization: The memory of each crow is then initialized. It is assumed that they have hidden their foods at their initial positions as the crows have no experiences yet:

$$
\text { mem }=\left[\begin{array}{lllll}
m_{1}^{1} & m_{1}^{1} \ldots \ldots \ldots . & m_{d}^{1} \\
m_{1}^{2} & m_{2}^{2} \ldots \ldots \ldots . & m_{d}^{2} \\
m_{1}^{F} & m_{2}^{F} \ldots \ldots \ldots . & m_{d}^{F}
\end{array}\right]
$$

5) Fitness function evaluation: For each crow, the quality of the crow position is determined by evaluating the fitness function with inserting the values of the decision variable.

6) New position generation: Crow (i) generates a new position by randomly selecting one of the flock crows (i.e crow (j)) and follows it to discover the position of the hidden food by the later one (mj ). The new position of crow (i) is obtained $x^{i, i t+1}=x^{i, i t}+r_{j} * f l^{i, i t}\left(m^{j, i t}-x^{i, i t}\right)$

where, $r j=$ is a random number in the range of $[0,1]$

7) New position feasibility checking: Crow new position feasibility is checked. If it is feasible, the crow updates its position. Otherwise, the crow stays in the same position.

8) The new positions fitness function evaluation: The fitness function value for each crow new position is determined.

9) Memory updating: The crows update their memory as follows:

$m^{i, i t+1}=x^{i, i t+1} * f \quad\left(x^{i, i t+1}\right)$

where, $\mathrm{f}$ is the fitness function value. Equation indicates that if the fitness function value of the new position of a crow is better than its memorized position value the crow updates its memory by the new position.

Termination criterion checking: Steps 5-8 are repeated until either it max or the optimal solution of the fitness function is reached. When the termination criterion is satisfied, the best position of the memory is specified as the optimization problem solution as shown in flowchart

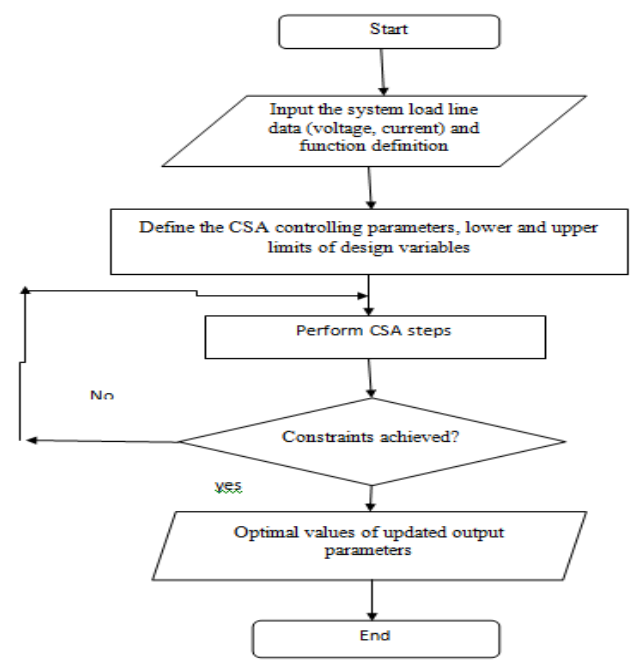

Fig.1 MF-CSA work flow chart

A single PV array block consist of 64 parallel strings where each string has 5 SunPower SPR-315E modules connected in series.

Each PV array is connected to a DC/DC converter (averaging) before finally providing a common DC input voltage for the inverter. Each boost is controlled by individual Maximum Power Point Trackers (MPPT). The MPPTs use the "Perturb and Observe" technique to vary the voltage across the terminals of the PV array in order get the maximum possible power. The final combination of DC output voltages from each string is taken as input DC voltage for inverter 
Here in this work we design a MF-CSA optimization controlled inverter for each phase which can be further integrated with the grid system.

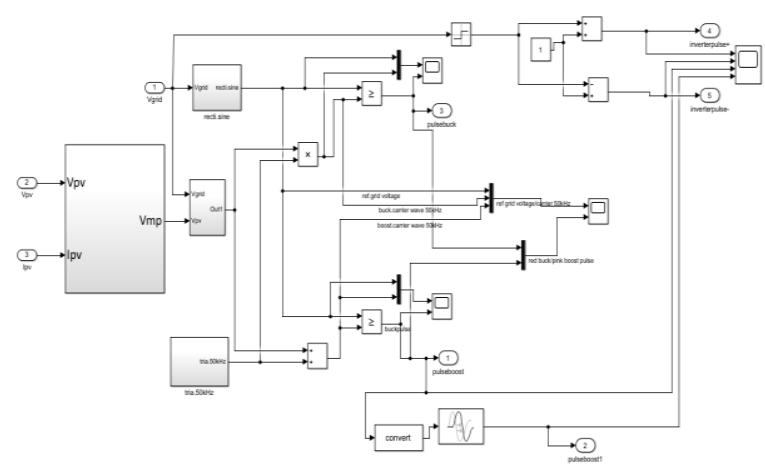

Fig. 2 Per phase converter pulse generation system implemented in the MATLAB/SIMULINK fed by MF-CSA

This control is optimized by using multifunction definition for the crow search optimization algorithm. The technique utilizes the power at the load line as optimizing equation for balancing its quality and adjusting according to the load changes. The sinusoidal waveform has been given as reference for monitoring the voltage and current waveform available at the loading point. The flow chart of the optimization algorithm has been shown in figure which is implemented in the MATLAB as governing equations and codes for generating pulses for per phase converter as well as the boosting pulses

\section{LOAD Analysis}

For stability study, to analyze the stability of a system including a power converter and constant power loads. We need to analyze the system stability from the ac side [6], [10]. The method considers a stability study for an AC and dc distribution system connected together via a power converter

Power systems supply power to loads that perform a function. These loads range from household appliances to industrial machinery. Most loads require a certain voltage and, for AC devices, a certain frequency and number of phases.

The analysis was performed for three different industrial loads. The results presented confirm the effectiveness of the proposed approach, which can be considered a very useful tool for the design and analysis of an electricity production system. MATLAB simulation was used to achieve the optimal configuration and size of the system components.

Table 1 shows the parameters that have been taken into account for the asymmetrical load. If the load is unbalanced, the lines / phases carry different currents and the total sum of these lines at the neutral point is not zero. The load in each phase is different and carries its own current. In this case, the neutral conducts the net asymmetrical current. For our analysis, we considered a resistive load of different sizes for each phase.

\begin{tabular}{|l|l|}
\hline \multicolumn{2}{|c|}{ Table 1: Parameters of unbalanced load } \\
\hline Resistive load of phase one (MW) & 0.1 \\
\hline Resistive load of phase two (MW) & 0.2 \\
\hline Resistive load of phase three (MW) & 0.3 \\
\hline
\end{tabular}

The three-phase dynamic load block implements a three-wire three-phase dynamic load whose active power $\mathrm{P}$ and reactive power Q vary as a function of the positive sequence voltage. The counter and zero currents are not simulated. The three load currents are therefore balanced even under asymmetrical load voltage conditions.

The impedance of the load is kept constant when the voltage at the terminals $\mathrm{V}$ of the load is lower than a certain value Vmin. When the voltage at the terminals is greater than the value Vmin, the active power $\mathrm{P}$ and the reactive power $\mathrm{Q}$ of the load vary as follows:

$$
\begin{aligned}
& P(s)=P_{0}\left(\frac{V}{V_{0}}\right)^{n_{p}} \frac{1+T_{p 1} s}{1+T_{p 2} s} \\
& Q(s)=Q_{0}\left(\frac{V}{V_{0}}\right)^{n_{q}} \frac{1+T_{q 1} s}{1+T_{q 2} s},
\end{aligned}
$$

\begin{tabular}{|l|l|}
\hline \multicolumn{2}{|c|}{ Table 2: Parameters of dynamic load } \\
\hline Active Power (MW) & 1 \\
\hline Reactive Power (KVar) & 0.2 \\
\hline Filtering time constant & $1 \mathrm{e}-3$ \\
\hline
\end{tabular}

\section{RESUlts}

The focuses research has been carried out in the following mentioned cases.

CASE 1: Solar PV distributed generation system designed with the converter having space vector selection control for each phase.

CASE 2: The solar PV distributed generation system with proposed per phase MF-CSA optimizing controller for converter.

The distortion level has been studied that concludes the quality output at the loading points. The distortion level has been carried by calculating the FFT (Fast Fourier Transform) of the voltage and current waveforms available at the load terminal. The system has been provided with the variable inputs namely irradiation and temperature at the solar panels offering the variation in the climatic factors at different regions. The THD calculation has been carried out for studying the initial quality issues with the output at the loading points. The following equation has been used to carry out the calculations: 
The finite, or discrete, Fourier transform of a complex vector with $\mathrm{n}$ elements is another complex vector $\mathrm{Y}$ with $\mathrm{n}$ elements:

$$
Y_{k}=\sum_{j=0}^{n-1} \omega^{j k} \mathcal{Y j}_{\mathrm{j}}
$$

Where $\omega$ is a complex nth root of unity:

$$
\omega=e^{-2 \pi i / n} .
$$

From the FT spectrum we can calculate THD (total harmonic distortion) of the given signal.

$$
\text { THD } \% \text { of fundamental }=\frac{\sqrt{\sum_{h=2}^{25} Q_{h}^{2}}}{Q_{1}} \times 100
$$

The chapter has dealt with the changes in the loading conditions as well by analysis the various loads such as balanced load, unbalanced load and dynamic load at the loading points. The various power outputs are described associated with each loads and the comparative improvement has been done.

CASE 1: Solar PV distributed generation system designed with the converter having space vector selection control for each phase.

The solar PV system has been modeled in this case in SIMULINK platform of the MATLAB software where the input parameters are being set in varying form to study the effects on the load line. The outputs from solar system are DC voltage that undergoes change to $\mathrm{AC}$ for by deploying the converters. The designing of converter has been achieved for each phase with respective controller for generating pulses that are intended to drive the IGBTs based converters. The pulse width modulation technique has been used for converters at each phase along with the space vector selection control and then it has been sent to the transformer that further integrates it grid energy system. The grid line voltage is $10 \mathrm{KV}$ line feeding various loads. The analysis has been carried out at this line to study the comparative results of the converter control.

The graphical output waveforms of voltage, current, active power and reactive power are shown in the figures below. Various loads are also driven to study the system efficiency and reliability.

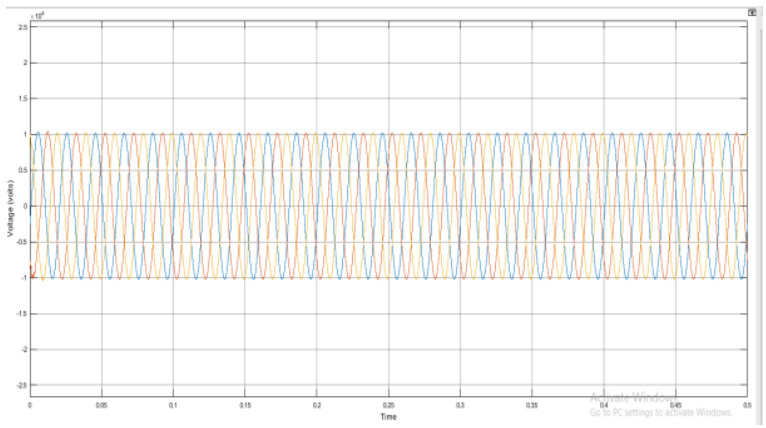

Fig. 3 Voltage available at the load point in system with converter having space vector selection control.

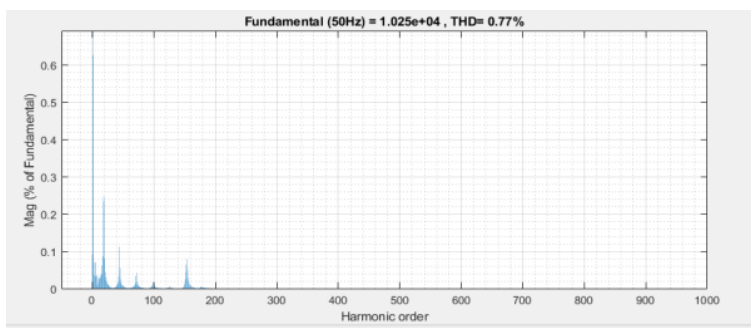

Fig. 4 THD\% calculation of voltage waveform in case1

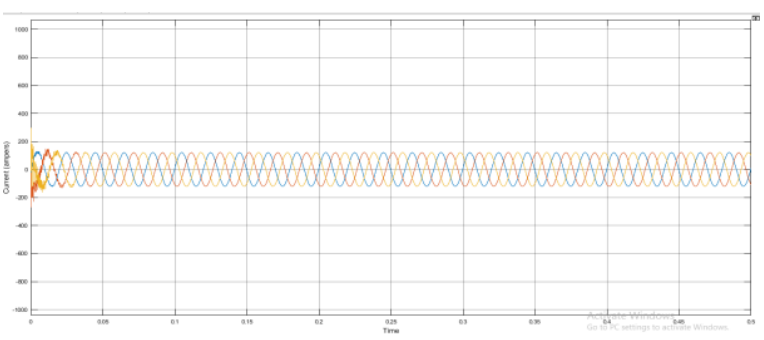

Fig. 5 Current drawn at the load point in system with converter having space vector selection control.

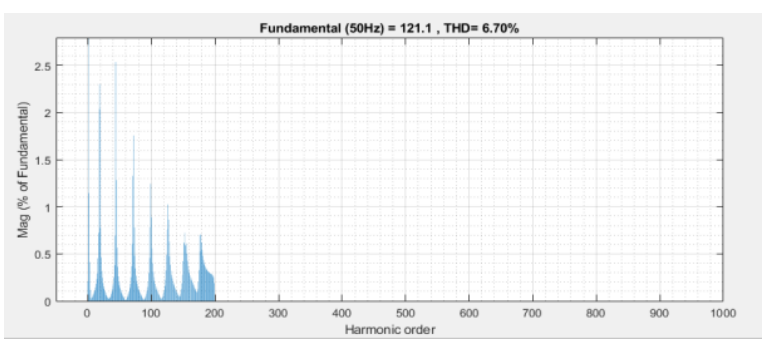

Fig. 6 THD\% calculation of the current in case 1

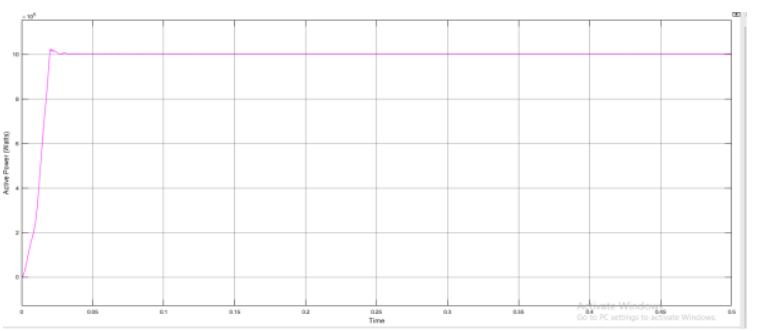

Fig. 7 Active Power available at the load point in system with converter having space vector selection control

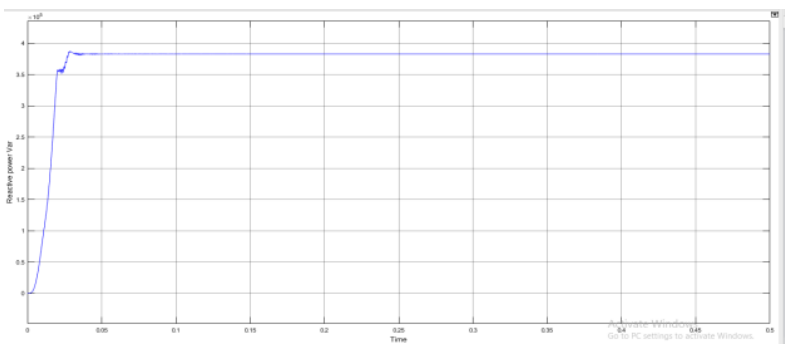

Fig. 8 Reactive Power available at the load point in system with converter having space vector selection control 
The above waveforms shows the voltage output, current output, active power output, and reactive power in system with converter having space vector selection PWM control. It is concluded that the voltage output is coming to be approximately 10 Kilo volts. The output available at the load line driving balanced load is found to approximately 121.1 Ampere with active power output as $1.003 \mathrm{MW}$ and reactive power output as $0.38 \mathrm{MV}$ ar.

CASE 2: The solar PV distributed generation system with proposed per phase MF-CSA optimizing controller for converter.

The converter designed for each phase has been controlled by utilizing AI based optimizing algorithm for the power quality enhancement and efficient load driving capability. The DC voltage output from the solar based system has been converted into the $\mathrm{AC}$ voltage using these converters. The aim is to maintain the grid voltage standard while attaining smooth changes in the current according to the load variations. The converter pulses are provided with $\mathrm{DC}$ and $\mathrm{AC}$ voltage references for optimization through MF-CSA optimizing controller. The converter is made to integrate the solar system to the three phase AC system. There has been designing of per phase inverter and its control while the DGs are fed with different kinds of loads at the high voltage line.

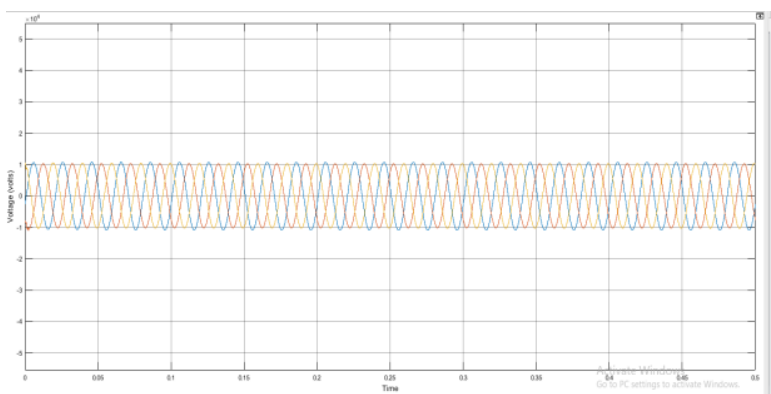

Fig.9 Voltage available at the load line in solar system having converters driven by per phase MF-CSA optimizing controller

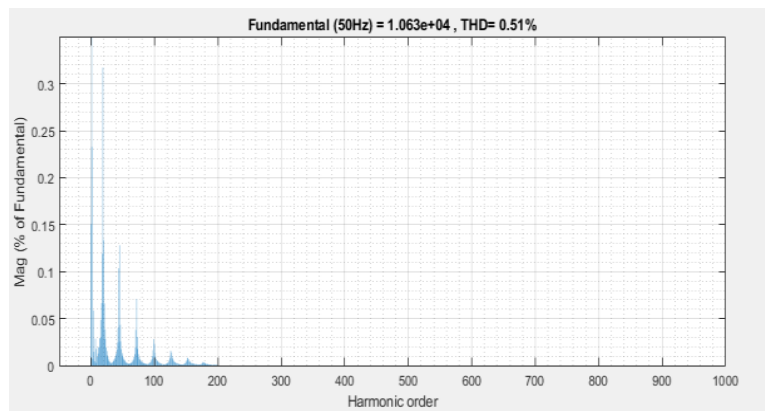

Fig. 10 THD $\%$ in line voltage in case 2

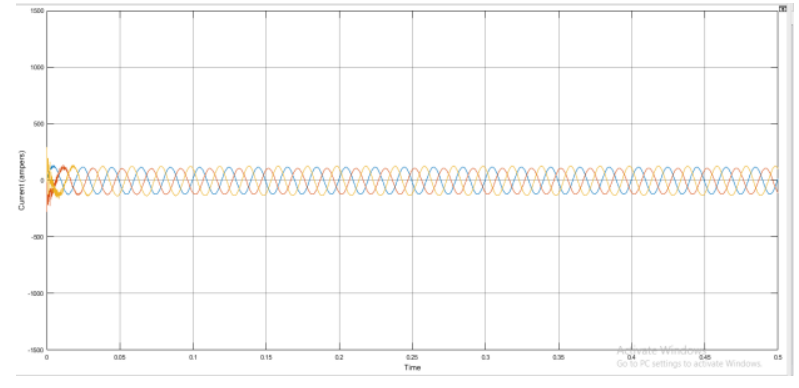

Fig. 11 Current drawn at the load line in solar system having converters driven by per phase MF-CSA optimizing controller

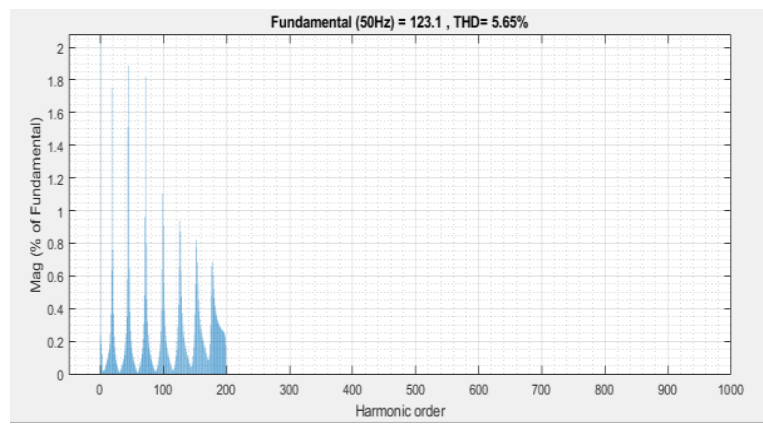

Fig. 12 THD\% in line current in case 2

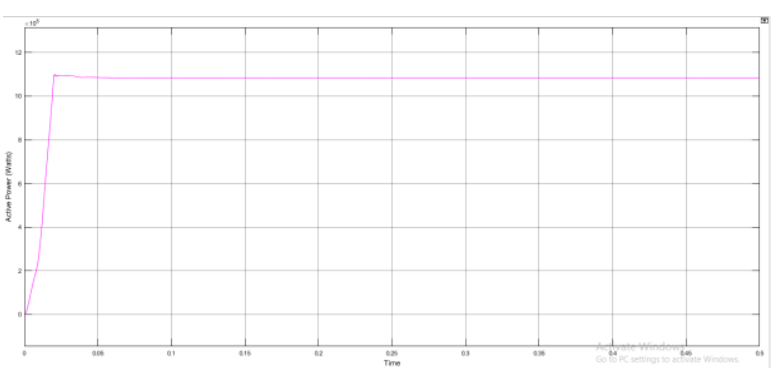

Fig. 13 Active power at the load line in solar system having converters driven by per phase MF-CSA optimizing controller

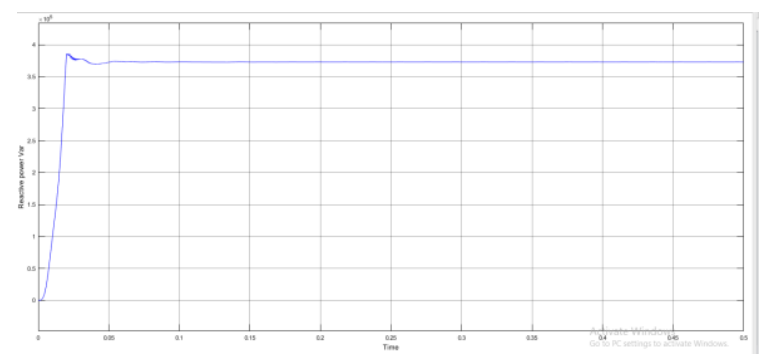

Fig. 14 Reactive power drawn at the load line in solar system having converters driven by per phase MF-CSA optimizing controller

In the system having proposed DG system power regulatory per phase inverter with proposed MF-CSA (Multi Function Crow Search Algorithm) the voltage output was maintained to be 10 Kilo volts for driving various loads. The current was found to be approximately 123.1 Ampere with active power output as $1.083 \mathrm{MW}$ and reactive power output as 0.37 MVar. A. Validation

The comparative results have been drawn out in terms of the harmonic distortion study, power enhancement and power 
factor correction as extracted from the study of the distributed generation based solar system whose converter designing is being achieved. The table 5.1 depicts the outcomes from the two systems being simulated in the SIMULINK environment. The percentage enhancement in terms of increase of decrease is also shown.

$\%$ increase in active power $=\frac{\text { new } A P-\text { old } A P}{\text { old } A P} \times 100$

$$
=\frac{1.083-1.003}{1.003} \times 100
$$

$=\operatorname{approx} 8 \%$

$\%$ decrease in reactive power $=\frac{\text { old } R P-\text { new } R P}{\text { old } R P} \times 100$

$$
=\frac{0.38-0.37}{0.37} \times 100
$$

$=\operatorname{approx} 2.7 \%$

\begin{tabular}{|l|c|c|c|}
\hline Parameters & $\begin{array}{c}\text { DG System } \\
\text { with } \\
\text { converter } \\
\text { having } \\
\text { space } \\
\text { vector } \\
\text { selection } \\
\text { control }\end{array}$ & $\begin{array}{c}\text { system } \\
\text { having } \\
\text { converters } \\
\text { driven by per } \\
\text { phase MF- } \\
\text { CSA } \\
\text { optimizing } \\
\text { controller }\end{array}$ & $\begin{array}{c}\text { Enhancement } \\
\text { done }\end{array}$ \\
\hline Voltage & $10 \mathrm{KV}$ & $10 \mathrm{KV}$ & Constant \\
\hline Current & 121.1 & 123.1 & 2 ampere \\
ampere & Ampere & $8 \%$ \\
\hline Active Power & $1.003 \mathrm{MW}$ & $1.083 \mathrm{MW}$ & reduced by \\
\hline THD \% in & $0.77 \%$ & $0.51 \%$ & 0.26 \\
voltage & $6.70 \%$ & $5.65 \%$ & $\begin{array}{c}\text { Reduced by } \\
1.05\end{array}$ \\
\hline $\begin{array}{l}\text { THD\% in } \\
\text { current }\end{array}$ & & & $\begin{array}{c}2.7 \% \\
\text { decreased }\end{array}$ \\
\hline $\begin{array}{l}\text { Reactive } \\
\text { Power }\end{array}$ & $0.38 \mathrm{MVar}$ & $0.37 \mathrm{Mvar}$ & $\begin{array}{c}\text { Increased by } \\
0.051\end{array}$ \\
\hline Power Factor & 0.828 & 0.879 & \\
\hline
\end{tabular}

It was found that active power output was considered to be enhanced from power of approximately $1.003 \mathrm{MW}$ to 1.083 MW and the voltage was kept constant to $10 \mathrm{KV}$. The objective was to keep the active power output stable and balanced which was obtained from the proposed controller having inverter with MF-CSA optimizing controller for each phase of the system. The reactive power was seen to be reduced.

B. Analysis of algorithm for various loads.

The work is further extended to analyze the algorithm of its efficiency in feeding the various kinds of loads. The two loads are being driven and the outcomes from them are studied namely the dynamic load and the unbalanced load. The analysis before has already covered the situations associated with the balanced loading conditions.

\section{MF-CSA algorithm feeding the unbalanced load}

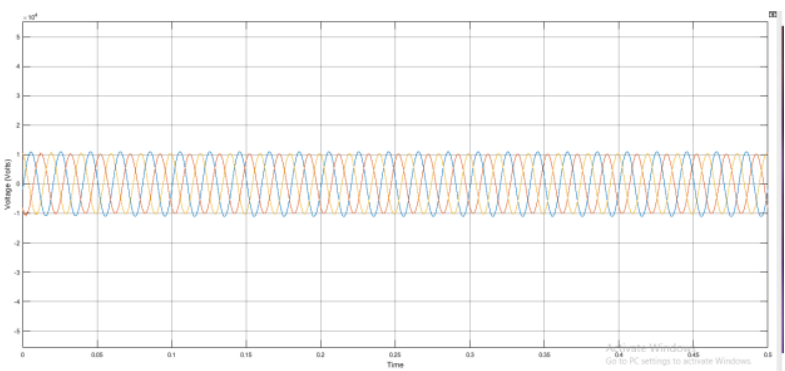

Fig. 15 Voltage available at the load line of unbalanced load

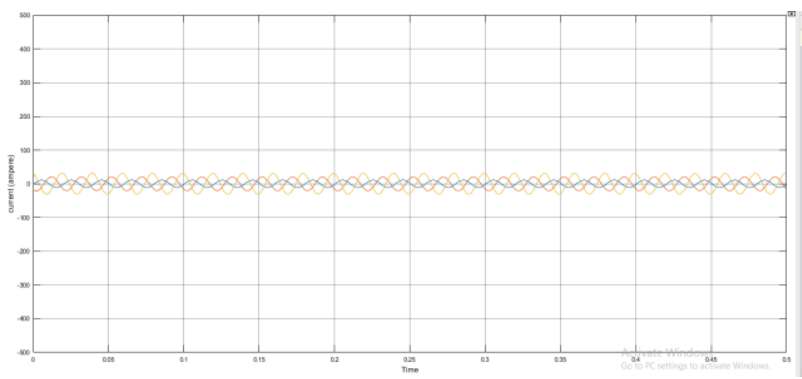

Fig. 16 Current available at the load line of unbalanced load

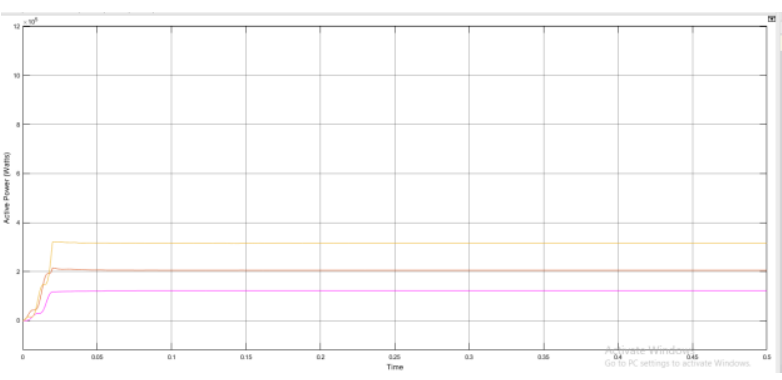

Fig. 17 Active Power at the load line of unbalanced load

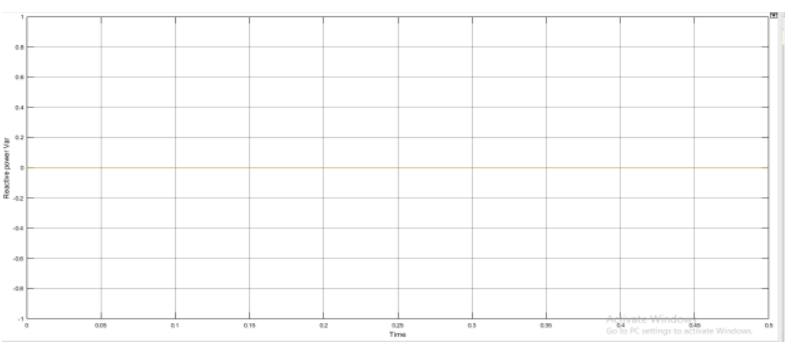

Fig. 18 Reactive Power at the load line of unbalanced load

The per phase loading in this condition is different. It was found that the power at each phase is stable and the voltage at the loading point is maintained to $10 \mathrm{KV}$. The current at each phase is different due to different values of resistive loads.

\section{MF-CSA algorithm feeding the dynamic load}




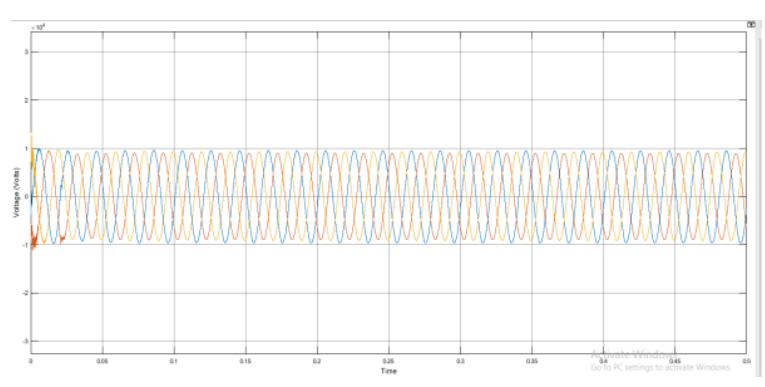

Fig. 19 Voltage in the load line with dynamic load

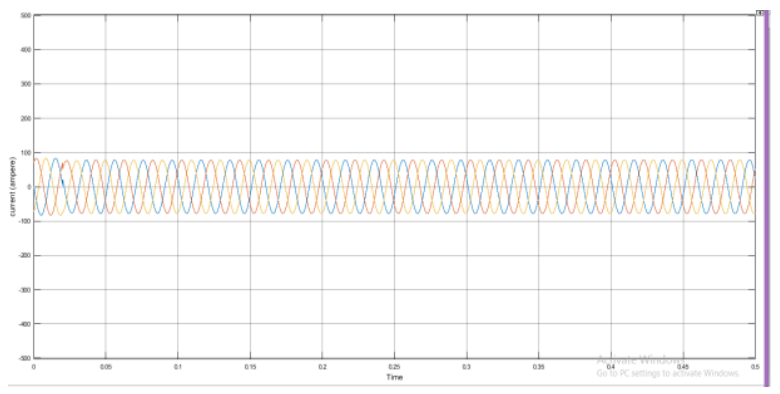

Fig. 20 Current at the load line with dynamic load

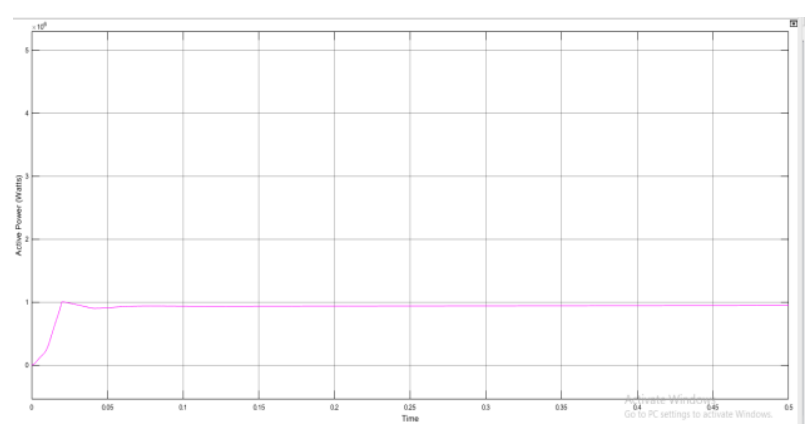

Fig. 21 Active Power at the load line with dynamic loading

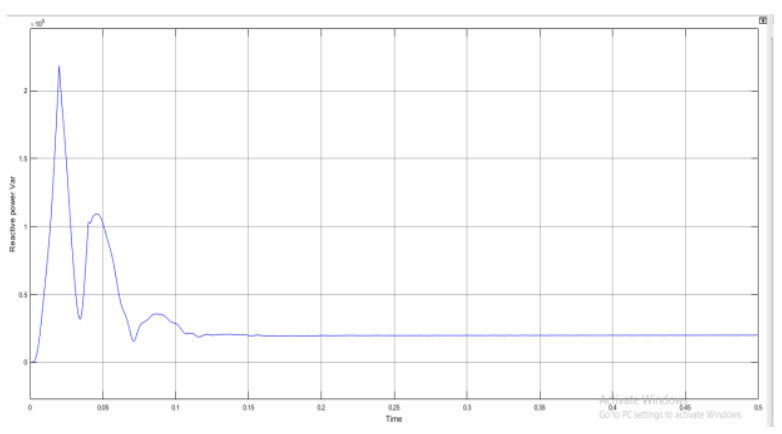

Fig. 22 Reactive Power at the load line with dynamic loading

\section{CONCLUSION}

This work provides a comprehensive design and implementation of power regulatory per phase inverter with proposed MF-CSA (Multi-Function Crow Search Algorithm) optimization controller. The Inverter has been provided with proposed optimization technique while integrating it with the grid. The voltage output of the system from the modeled solar system with varying irradiation and temperature control is being fed to the inverter for $\mathrm{DC}$ to $\mathrm{AC}$ conversion. This voltage is then fed to the grid. The grid voltage being maintained constant to $10 \mathrm{KV}$ in both the systems

- The system designed with this as seen considerable improvement in active power output from the system from approximately $1.003 \mathrm{MW}$ in conventional inverter to $1.083 \mathrm{MW}$ in the proposed scheme.

- The proposed system is also made to feed unbalanced load for the different phases and dynamic load to measure its capability of driving various loads.

- The THD\% in both voltage and current waveforms have been reduced while using power regulatory per phase inverter with proposed differential evolutionary pulse regulation control

- $\quad$ Finally the work is made efficient y integrating it with grid. The designed system is also capable of feeding reactive power to the grid when required.

The computational methodology of the proposed modulation technique is very easy and the technique can be applied to multilevel inverter with any number of levels.

\section{VII.FUTURE SCOPE}

The proposed controller was seen to efficiently balance the active as well as reactive power using the optimization control during the load switching conditions. However the stabilization time of reactive power was more as compared to active power. The controller can be in future modeled for the stabilization time reduction in reactive power as well.

By connecting the super capacitor to the link of an inverter the converter can be designed for a higher voltage and a topology with transformer has to be used. Also the inverter control can be improved employing certain changes in the SPVM such that it can handle balanced and unbalanced DC voltages

The work can further be extended to the hybrid system such that the controller is redesigned to accommodate for changes in both solar input and wind energy system input. The controller has to adjust changes in variation in wind speed as well

\section{REFERENCE}

[1] Darshni M. Shukla, Naimish Zaveri "Performance of Three Level H-Bridge Inverter in Grid Connected Solar PV for Multifunctional Operations using Different Control Techniques" International Journal of Innovative Technology and Exploring Engineering (IJITEE), Volume-9 Issue-4, February 2020.

[2] S. Balasubramanyan and M. Sasirekha "Optimization Strategy for Reactive Power Compensation in Grid Connected PV Systems" Middle-East Journal of Scientific Research 24 (3): 892-897, 2016.

[3] P M Ansho "A Novel Method for Reactive Power Management For Grid Connected Cascaded Photovoltaic Systems" International Journal of Advanced Research in Management, Architecture, 
Technology and Engineering (IJARMATE) Vol. 2, Special Issue 11, March 2016.

[4] Kangan Wang, Xiaojie Wu "A Dynamic Power Distribution Strategy for Large-scale Cascaded Photovoltaic Systems" Journal of Power Electronics, Vol. 17, No. 5, pp. 1317-1326, September 2017.

[5] Hee-Jun Kim "Special sample and hold techniques", National Semiconductor, Application Report 294. Available: http://www.national.com/an/AN/AN294.pdf

[6] Seyed mahmoudian, M.; Rahmani, R.; Mekhilef, S.; MaungThanOo, A.; Stojcevski, A.; Soon, TeyKok; Ghandhari, A. S. (2015-07-01). "Simulation and Hardware Implementation of New Maximum Power Point Tracking Technique for Partially Shaded PV System Using Hybrid DEPSO Method". IEEE Transactions on Sustainable Energy. 6 (3): 850-862. doi:10.1109/TSTE.2015.2413359. ISSN 1949-3029.

[7] Sonali Surawdhaniwar, Diwan, Ritesh (July 2012). "Study of Maximum Power Point Tracking Using Perturb and Observe Method". International Journal of Advanced Research in Computer Engineering \& Technology. 1 (5): 106-110.

[8] S. Thirumaliah, A.P.Yadav and G. Harith. "Comparison of MPPT Algorithms for DC-DC Converters Based PV Systems" International Journal of Advanced Research in Electrical, Electronics and Instrumentation Engineering Vol. 1, Issue 1, July 2012.

[9] Y.-H.Chang and C.-Y. Chang, "A Maximum Power Point Tracking of PV System by Scaling Fuzzy Control," presented at International Multi Conference of Engineers and Computer Scientists, Hong Kong, 2010.

[10] Zainuri, Pooja Singh, Radzi, M.A.M., Soh, A.C., et al.: 'Development of adaptive perturb and observe-fuzzy control maximum power point tracking for photovoltaic boost dc-dc converter', IET Renew. Power Gener., 2014, 8, (2), pp. 183-194. 